\title{
Statistical Forecasting for Offshore Wind Helicopter Operations
}

\author{
José Antonio Domínguez- \\ Navarro \\ University of Zaragoza \\ Spain \\ jadona@unizar.es
}

\author{
Iain Dinwoodie \\ Institute for Energy \& Environment \\ University of Strathclyde \\ Glasgow, UK \\ iain.dinwoodie@strath.ac.uk
}

\author{
David McMillan \\ Institute for Energy \& Environment \\ University of Strathclyde \\ Glasgow, UK \\ dmcmillan@eee.strath.ac.uk
}

\begin{abstract}
The influence of the wind and wave climate on offshore wind operations and maintenance is well known. These environmental factors dictate to a large extent whether turbine crew transfer (carried out by small vessels) or major lifting actions (carried out by large vessels) can be executed at sea. However the role of helicopter operations has received much less attention. In this paper the authors explore the helicopter access problem via statistical forecasting and implement a model innovation, by including cloud base as a key access metric. By understanding the practical limits of helicopter operation, offshore wind access calculations will be much improved and reflect more closely the reality of operations at sea.
\end{abstract}

Keywords- Wind Turbine; Helicopter Operations; Statistical forecasting;ARMA; Markov Chain; Fuzzy Logic; Wavelets.

\section{INTRODUCTION}

The need to understand and control the costs of offshore wind is well known. A key aspect of the problem is operations and maintenance, contributing roughly $25 \%$ of the total cost of energy. In order to make better cost estimates, it is imperative that the practicalities of operation are captured in modeling tools. Helicopter operations have a key role to play in offshore operations as an augment to small crew transfer vessels for maintenance tasks not requiring heavy lift operations [1]. This paper therefore focuses on bringing together 3 key variables (wind speed, wave height, and cloud cover) in order to provide more realistic estimates of accessibility for helicopter operations.

\section{PREVIOUS WORK}

Early work on offshore wind farm maintenance and access modeling can be traced to the late 1990's and the research of van Bussel et al. [2] and Rademakers et al. [3]. Many authors since then have attempted to analyse the installation, maintenance and logistics problem - such as Walker et al. [4]. One aspect of this problem which has not received much attention to date is helicopter access. Helicopter access has historically been crudely modeled as being constrained by wind speed only, or a mixture of wind speed and wave height. This paper moves a step beyond this by considering the impact of visibility - in the form of cloud cover - and its impact on helicopter access. For the purposes of this paper, this is considered as a short term forecasting and scheduling problem. Data from the North Sea are used in a case study comparing accessibility forecasts using a trio of different statistical methods.

\section{DATA}

The data used in this paper is derived from the British Atmospheric Data Centre's MIDAS marine surface measurements [5]. The data set numbers over 100 measurement locations on the seas around the UK. However the data quality is highly variable and a filtering procedure had to be developed to identify suitable sites. The result of this quality checking can be seen in Figure 1 . The northern location of Sedco 711 (an oil rig drilling unit at the times of measurement operating in the North Sea) and Morecambe bay in the Irish Sea were selected as being the only two sites both with adequate data coverage (equivalent to 7 years with effective time resolution of 3 hours) and a location broadly representative of wind and wave conditions encountered at offshore wind sites. Figure 2 illustrates the geographical location of each site. Gaps in data were filled using a cubic interpolation between points. The consequence of data quality and this simplistic gap filling approach is discussed in the results section of this paper.

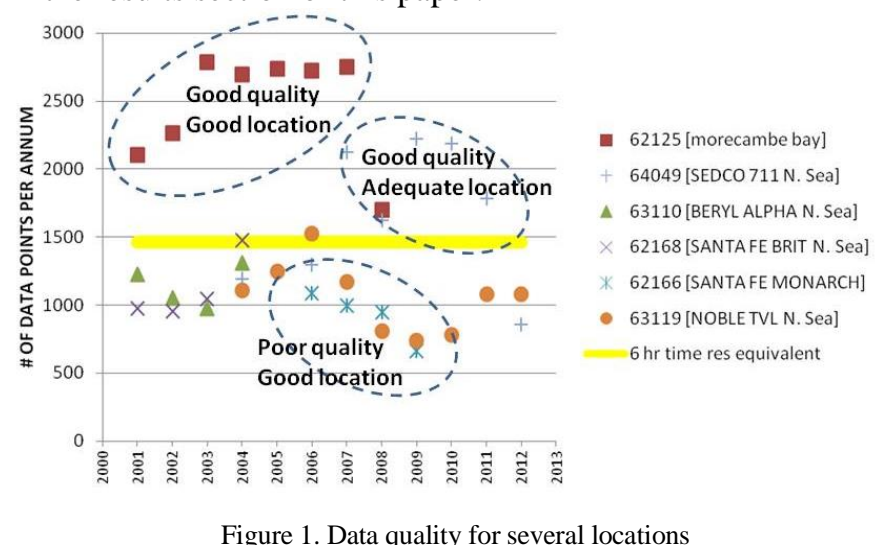

Figure 1. Data quality for several locations 


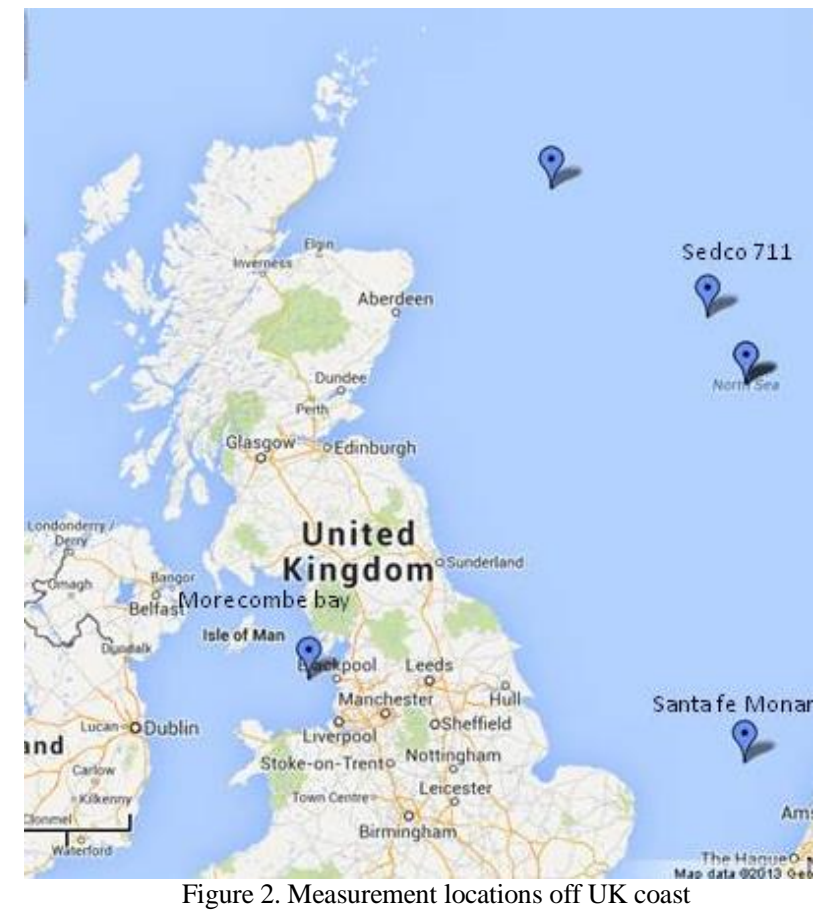

IV. FORECASTING MODELS

\section{A. ARMA}

Auto-Regressive approaches to describe time series data were originally developed in [6], and have since been applied to a diverse range of applications. Of particular relevance to this work, AR models have been used to describe significant wave height [7], mean wind speeds for wind turbine power generation [8] and wind turbine maintenance [9]. The AR model, normalized to the mean of the data is described in Eq. (1).

$$
X_{t}=\mu+\varepsilon_{t}+\sum_{i=1}^{p} \varphi_{i}\left(X_{t-i}-\mu\right)
$$

The model described in Eq. (1) is valid only for a normally distributed process. Neither annual wind speed nor significant wave heights follow a normal distribution and must therefore be transformed before Eq. (1) is applied to the data sets.

For significant wave heights it is necessary to remove the fit of monthly mean and then apply a Box-Cox transformation on the data shown in Eq. (2) [7].

$$
Y_{t}=T\left(H s_{t}\right)=\ln \left(H s_{t}\right)-\hat{\mu}_{\ln \left(H s_{t}\right)}
$$

The required order of AR model was determined using the auto-correlation function and partial autocorrelation function and determined as 4 for wave models. The determination of AR coefficients and model generation was performed using the MATLAB system identification toolbox

\section{B. Markov Chain}

Markov Chains have been deployed to solve several problems in the wind energy literature. Sayas \& Allan [10] used this framework to model wind turbine failure rates and the influence of wind speed on reliability. In terms of forecasting applications, the work of Pinson and Madsen is prominent [11]

This paper applies a pure Markov chain (that is memoryless and time-homogenous parameters) with discrete time (dt $=3$ hours) and discrete state space to model and forecast wind, wave and cloud cover data. The main criteria when setting up the chain is the bin size, which determines how the state space is partitioned. This is established first by determining the maximum value in the data set. Then an appropriate bin size is chosen, which is specific to the variable being modeled (for example, the modeler should take into account the resolution of the original data when selecting the bin size). This process is illustrated in Table I. In this way the Markov chain states map to the physical variable.

TABLE I. TYPICAL PARAMETER VALUES (MARKOV CHAIN)

\begin{tabular}{l|ccc} 
& Wind speed (kts) & Sig. wave ht $(\mathrm{m})$ & Cloud base (dec) \\
\hline Maximum value & 35.0 & 11.0 & 250.0 \\
Bin size & 1.0 & 0.05 & 5.0 \\
Number of states & 36 & 221 & 51
\end{tabular}

The parameter estimation process is based on the normalized frequency of transition from one state to another (eg state a to $\mathrm{b}$, in time step $\mathrm{k}$ ), and the frequency balance method in [12] and is summarized below.

$$
\mathrm{P}_{\mathrm{a}, \mathrm{b}}=\mathrm{P}\left(\mathrm{s}_{\mathrm{b}}, \mathrm{t}_{\mathrm{k}+1} \mid \mathrm{s}_{\mathrm{a}}, \mathrm{t}_{\mathrm{k}}\right) \mathrm{k}=1,2,3 \ldots \mathrm{N}
$$

\section{Wavelets/Neural Network}

A problem of all forecasting methods is that the quality of input data influences in the accuracy of the prediction. A solution is to use a filter to eliminate the uncertainty of high frequency. This method [10] has two phases: Filter phase and forecasting phase.

\section{a) Filter phase}

A signal $\mathrm{f}(\mathrm{t})$ can write as an "approximation" plus "details" (4) according to wavelet analysis [11].

$$
f(t)=\sum_{k} c_{j 0}(k) \varphi_{j 0, k}(t)+\sum_{k} \sum_{j=j 0}^{J-1} d_{j}(k) \psi_{j, k}(t)
$$

The coefficients $c_{\mathrm{j}}(k)$ and $d j(k)$ that multiple to functions $\varphi_{\mathrm{j} 0, \mathrm{k}}(t)$ and $\psi_{\mathrm{j}, \mathrm{k}}(t)$ can be calculated with a digital filter, Fig. 3 . 


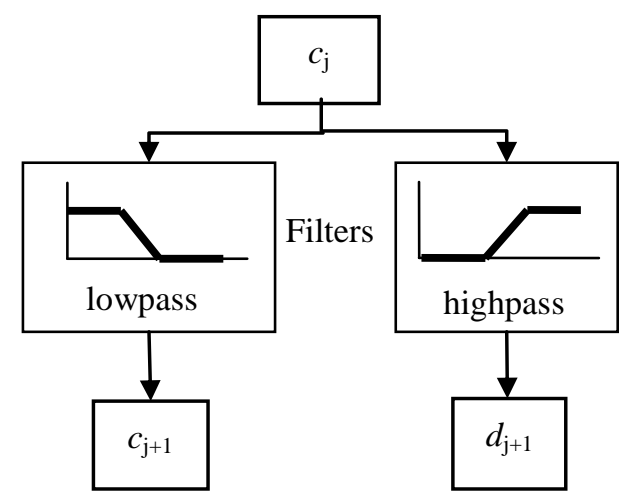

Figure 3. Signal decomposition

The "details" are neglected and forecasting is made only on the "approximation".

\section{b) Forecasting phase}

In this phase, a method based on neural network [12] is used to obtain the prediction of the signal. In this case, a multilayer perceptron find the nonlinear relations in the time series:

$$
y_{t}=\sum_{j} w^{\prime}{ }_{k j} g\left(\sum_{i}\left(w_{j i} y_{t-i}-\theta_{j}\right)-\theta^{\prime}{ }_{k}\right)
$$

where $\theta_{\mathrm{j}}$ and $\theta_{\mathrm{k}}$ are layers thresholds, and $w_{\mathrm{ji}}$ and $w_{\mathrm{kj}}$ are layers weights, $i$ and $j$ are the number of neurons in each layer, $g$ is the activation function.

\section{RESUlTS}

\section{A. Model Testing}

Each of the forecasting models previously described was tested on the available data sets to allow their performance to be benchmarked. The model parameters were fitted using a training period and then forecasts generated at 3,6 and 9 hour look-ahead periods over the remaining duration of the time series. For the North Sea location the training period consisted of years 2007-2009 and forecasting period of 2007-2010. For the Morecombe Bay location the training period consisted of years 2002-2006 and forecasting period of 2007-2008. In addition, to the described model, persistence forecast was performed where the forecast value is equal to the last observation. This represents the simplest forecasting approach and provides a baseline with which to compare the more advanced modeling approaches.

\section{B. Forecasting Benchmark}

The standard forecasting accuracy metric, Root Mean Square Error (RMSE) was initially used in order to give an indication of the forecasting performance of each model on different climate data sets. The benchmark results of all cases are shown in Figures 4 to 8.

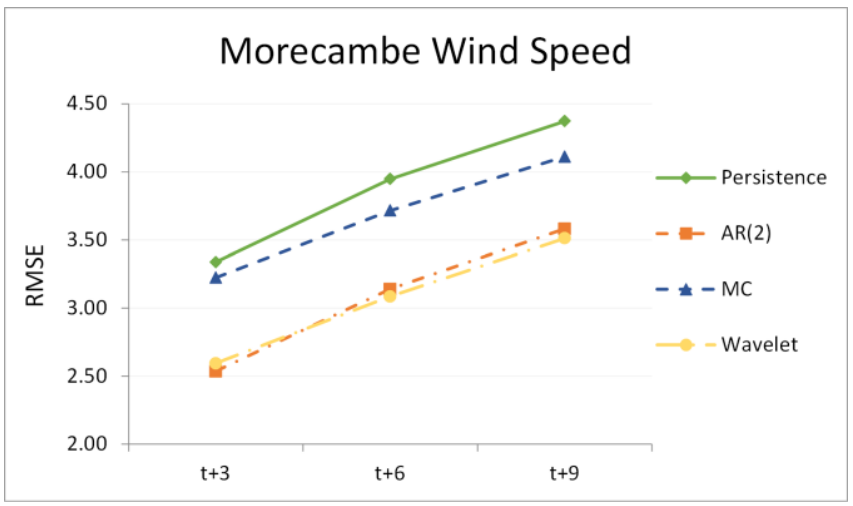

Figure 4. RMSE for Morecambe Bay Wind Speed

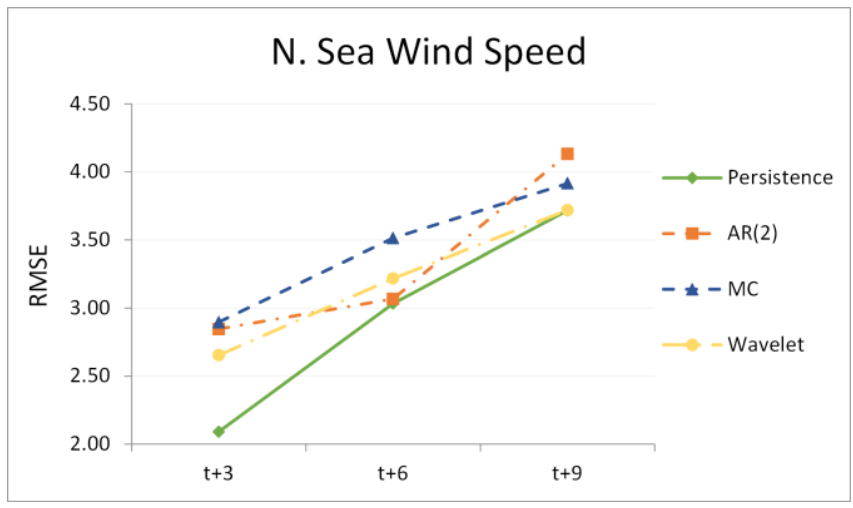

Figure 5. RMSE for North Sea Wind Speed

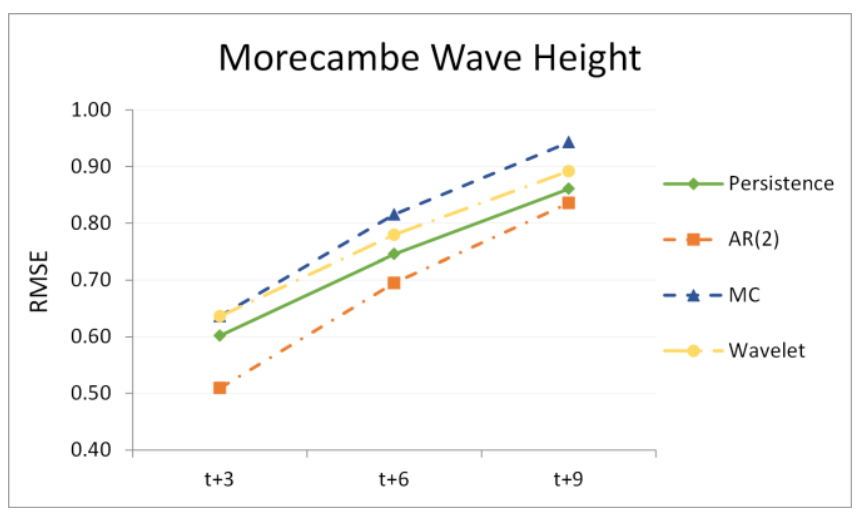

Figure 6. RMSE for Morecambe Bay Significant Wave Height

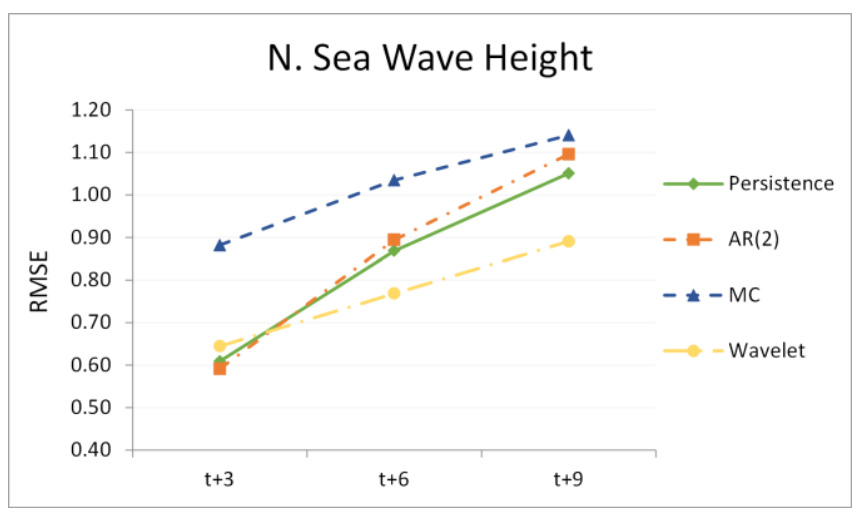

Figure 7. RMSE for North Sea Significant Wave Height 


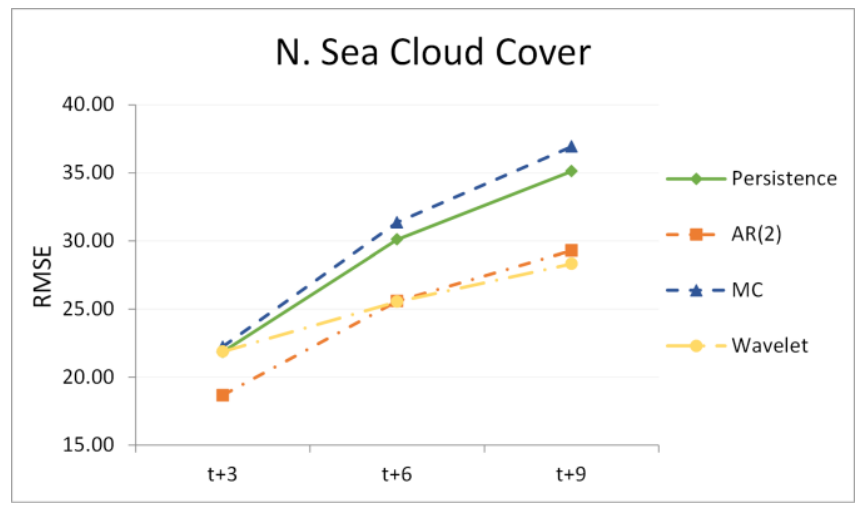

Figure 8. RMSE for Morecambe Cloud Cover Level

The results show that the probabilistic models presented are capable of reducing forecast error when compared to a baseline persistence model in most cases. However, the performance of the models is shown to be sensitive to the quality of the training and forecast data, in particular for significant wave height time series. Considering Figures 4 and 5 all models outperform persistence when there is good quality data in the Morecambe Bay site with the ARMA and Wavelet models performing best. However, when the data quality is poorer, the simpler persistence model outperforms the more complex models. This is attributed to the simplistic cubic spline approach adopted to gap filling which results in a gradual change in value leading to small errors when compared to the preceding time step. The Wavelet model appears most robust to dealing with poor quality data. This is also evident from Figures 7 and 8 which also correspond to the North Sea data set where the Wavelet model performs best. The ARMA model outperforms all other models for the high quality wave time series and this is attributed to ability of the model to preserve the strong auto-correlation characteristics observed between time steps in the wave data. The Markov Chain forecast only improves on persistence for the case when high quality wind speed data is available suggesting it is a less robust model.

\section{Helicopter Access Forecast Metric}

RMSE is a useful metric, as it provides a general indication of forecast accuracy. However the value of a forecast may not be fully understood by RMSE benchmarking. Understanding the decision that should be supported by the forecast is crucial in establishing the overall value of a forecast to the end user. Access is determined based on the three climate characteristics being below the threshold values in Table II which are representative of current operational practices for offshore wind.
TABLE II. Typical Parameter VAlues (Markov Chain)

\begin{tabular}{l|c}
\hline Variable & Typical Values \\
\hline Significant Wave Height & $1.5 \mathrm{~m}$ \\
Wind Speed & $10 \mathrm{~m} / \mathrm{s}(\sim 19.5 \mathrm{kts})$ \\
Cloud Level & 30 decameters $(\sim 1000$ feet $)$ \\
\hline
\end{tabular}

For helicopter operations, there are two general implications of a bad forecast.

- False positive $(\mathrm{F}+)$ Forecast. Helicopter is dispatched but weather does not allow operation to be completed. Potentially, this impacts on fuel cost.

- $\quad$ False negative $(\mathrm{F}-)$ Forecast. Helicopter is not dispatched however a weather window does exist. This impacts lost energy from the wind turbine.

These metrics (shown in Fig. 9) were used to benchmark the forecasters with respect the 3 individual variables at Morecambe bay, and also to do a comparison in which all 3 variables are combined to come to a helicopter access decision.

Inspection of Fig. 9 shows that the performance of the forecasters is dependent on the variable in question, and that performance in relation to decision making also varies widely in terms of quality. F+ and F- forecasts can vary widely for the same variable at the same time horizon. Of particular interest is a comparison of the panel in Fig. 9 with the RMSE plots for the same site (Figs 5, 7, 8). In several of the RMSE plots, the forecasters outperform persistence. However, when the actual decision to be supported in factored in to the evaluation, as in Fig. 9, it appears that persistence is the best solution.

This illustrates the importance in forecasting of understanding the decision supported by the forecast. Benchmarking on metrics which do not necessarily relate to the decision, such as RMSE, may not yield the best results for the end-user - in this case and offshore wind farm operator, whose crew dispatch decisions drive O\&M costs. 

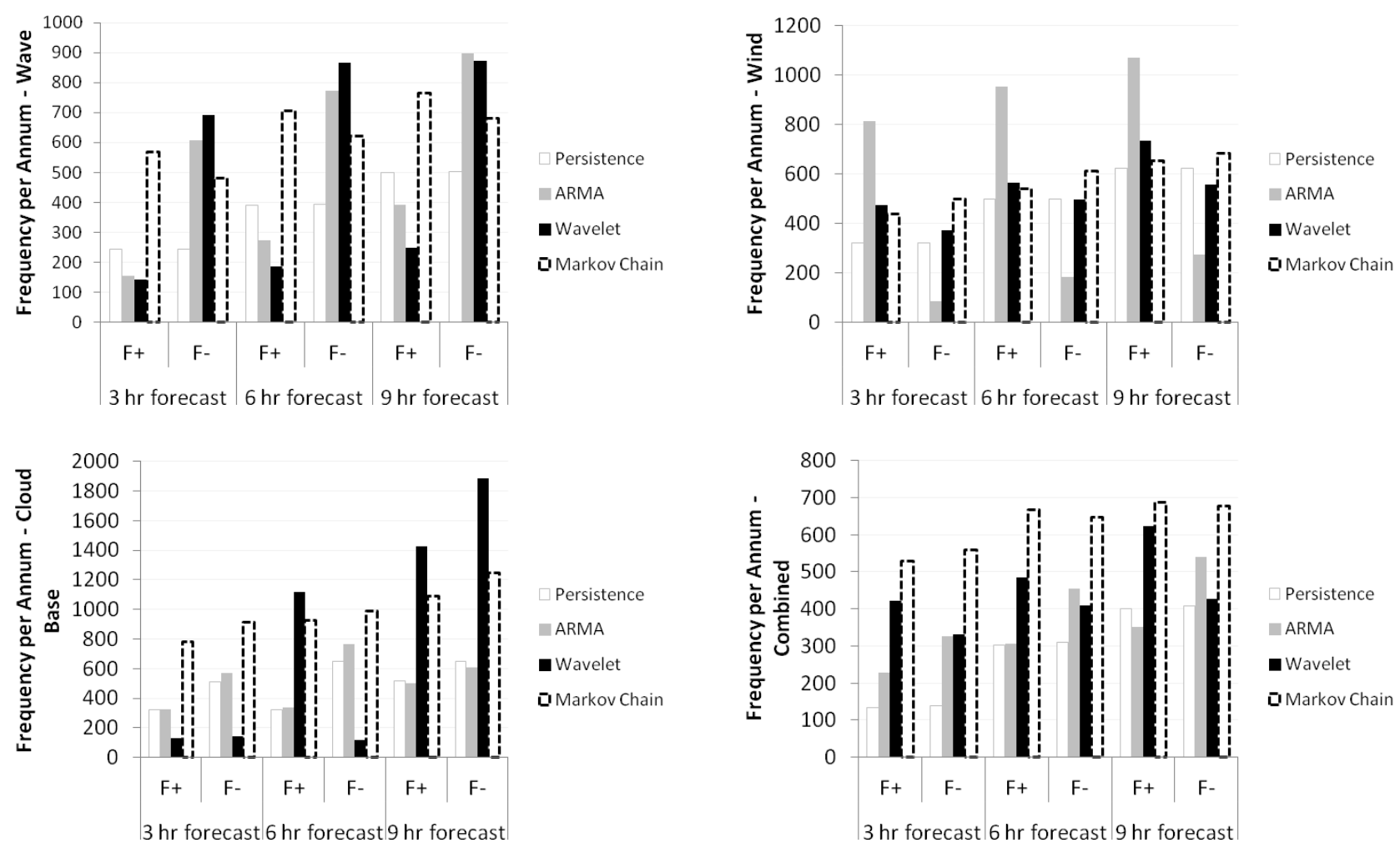

Figure 9. Panels show F+ and F- for each variable, and the combined forecast

\section{CONCLUSIONS}

A variety of short term forecasting methods have been applied to the area of helicopter accessibility for offshore wind turbine maintenance. Considering standard metrics an improvement over a basic persistence approach have been demonstrated for the three key accessibility metrics, wave height, wind speed and visibility via cloud cover. The improvement offered over persistence is sensitive to data quality, with little improvement or poorer forecasting performance observed for poorer quality data sets. This is of particular importance in the offshore environment where collection of data is expensive and prone to large periods of downtime.

The limitations of using error metrics when considering the impact of forecasting accuracy have been identified. Consequently the ability of the models to correctly predict operations based on access thresholds has been investigated for each access parameter and for a combined forecast. The simple statistical forecasting models presented in this paper fail to provide an improvement over the baseline persistence model. Exploration of more sophisticated but computationally demanding simulation models may provide improved performance and have been identified for future work. An investigation into the capabilities of the models with more complete data sets or using more sophisticated gap filling approaches has been identified as an area for future research.

\section{ACKNOWLEDGEMENTS}

The authors are grateful to the British atmospheric data centre (badc.nerc.ac.uk) for use of their data and interpretation of key data quality metrics. 
[1] I. Dinwoodie and D. McMillan, "Heavy Lift Vessel Strategy Analysis for Offshore Wind," presented at the European Wind Energy Association (EWEA) Conference, Vienna, 2013.

[2] G. J. W. van Bussel and C. Schöntag, "Operation and Maintenance Aspects of Large Offshore Windfarms," presented at the European Wind Energy Conference EWEC 1997.

[3] L. W. M. M. Rademakers, H. Braam, M. B. Zaaijer, and G. J. W. vanBussel, "Assessment and optimisation of operation and maintenance of offshore wind turbines," presented at the European Wind Energy Conference EWEC, Madrid, 2003.

[4] R. T. Walker, J. van Nieuwkoop-McCall, L. Johanning, and R. J. Parkinson, "Calculating weather windows: Application to transit, installation and the implications on deployment success," Ocean Engineering, vol. 68, pp. 88-101, 2013.

[5] BADC, "MIDAS Marine surface measurements," ed.

[6] G. E. P. Box and G. M. Jenkins, Time series analysis : forecasting and control. San Francisco ; London: Holden-Day ; [Maidenhead] : Distributed by McGraw-Hill, 1970.

[7] C. G. Soares, A. M. Ferreira, and C. Cunha, "Linear models of the time series of significant wave height on the Southwest Coast of Portugal," Coastal Engineering, vol. 29, pp. 149-167, Dec 1996.

[8] D. Hill, D. McMillan, K. Bell, D. Infield, and G. W. Ault, "Application of Statistical Wind Models for System Impacts," Upec: 2009 44th International Universities Power Engineering Conference, pp. 761-765 1004, 2009.

[9] D. McMillan and G. W. Ault, "Condition monitoring benefit for onshore wind turbines: sensitivity to operational parameters," Iet Renewable Power Generation, vol. 2, pp. 60-72, Mar 2008.

[10] F. C. Sayas and R. N. Allan, "Generation availability assessment of wind farms," IEE Transactions on Generation Transmission Distribution, vol. 143, pp. 507-518, 1996.

[11] P. Pinson and H. Madsen, "Adaptive Modelling and Forecasting of Offshore Wind Power Fluctuations with Markov-Switching Autoregressive Models," Journal of Forecasting, vol. 31, pp. 281-313, 2012.

[12] R. Billinton and R. N. Allan, Reliability of Engineering Systems 2nd ed.: Plenium Press, 1992. 